\title{
Electrocorticographic Recording of Cerebral Cortex Areas Manipulated Using an Adeno-Associated Virus Targeting Cofilin in Mice
}

\author{
Julien Dufort-Gervais ${ }^{1}$, Robbert Havekes ${ }^{2}$, Valérie Mongrain ${ }^{1,3}$ \\ ${ }^{1}$ Center for Advanced Research in Sleep Medicine, Recherche CIUSSS-NIM ${ }^{2}$ GELIFES, University of Groningen ${ }^{3}$ Department of Neuroscience, Université \\ de Montréal
}

\section{Corresponding Author}

Valérie Mongrain

valerie.mongrain@umontreal.ca

\section{Citation}

Dufort-Gervais, J., Havekes, R. Mongrain, V. Electrocorticographic Recording of Cerebral Cortex Areas Manipulated Using an Adeno-Associated Virus Targeting Cofilin in Mice. J. Vis. Exp. (168), e61976, doi:10.3791/61976 (2021).

\section{Date Published}

February 21, 2021

DOI

$10.3791 / 61976$

URL

jove.com/video/61976

\section{Abstract}

The use of electrocorticographic (ECoG) recordings in rodents is relevant to sleep research and to the study of a wide range of neurological conditions. Adeno-associated viruses (AAVs) are increasingly used to improve understanding of brain circuits and their functions. The AAV-mediated manipulation of specific cell populations and/ or of precise molecular components has been tremendously useful to identify new sleep regulatory circuits/molecules and key proteins contributing to the adverse effects of sleep loss. For instance, inhibiting activity of the filamentous actin-severing protein cofilin using AAV prevents sleep deprivation-induced memory impairment. Here, a protocol is described that combines the manipulation of cofilin function in a cerebral cortex area with the recording of ECoG activity to examine whether cortical cofilin modulates the wakefulness and sleep ECoG signals. AAV injection is performed during the same surgical procedure as the implantation of ECoG and electromyographic (EMG) electrodes in adult male and female mice. Mice are anesthetized, and their heads are shaved. After skin cleaning and incision, stereotaxic coordinates of the motor cortex are determined, and the skull is pierced at this location. A cannula prefilled with an AAV expressing cofilin ${ }^{S 3 D}$, an inactive form of cofilin, is slowly positioned in the cortical tissue. After AAV infusion, gold-covered screws (ECoG electrodes) are screwed through the skull and cemented to the skull with gold wires inserted in the neck muscles (EMG electrodes). The animals are allowed three weeks to recover and to ensure sufficient expression of cofilin ${ }^{\mathrm{S} 3 \mathrm{D}}$. The infected area and cell type are verified using immunohistochemistry, and the ECoG is analyzed using visual identification of vigilance states and spectral analysis. In summary, this combined methodological approach allows the investigation of the precise contribution 
of molecular components regulating neuronal morphology and connectivity to the regulation of synchronized cerebral cortex activity during wakefulness and sleep.

\section{Introduction}

Electroencephalographic (or generally electrocorticographic [ECoG] in rodents) and electromyographic (EMG) recordings are extensively used in sleep research as well as more broadly in neuroscience, neurology, and psychiatry. In combination, these electrophysiological signals allow for the identification of vigilance states and the subsequent quantification of state duration and spectral composition, both in humans and rodents ${ }^{1,2,3,4}$. Such quantification has been useful to understand how sleep is modified in pathological conditions such as neurodegenerative diseases and models ${ }^{5,6,7}$ or by genetic modification ${ }^{8,9}$. For instance, the knockout (KO) of different genes linked to neuronal communication was shown to change the duration of wakefulness and sleep in both the mouse and fruit fly $10,11,12,13$. To tackle potential developmental compensation arising from the study of full-body $\mathrm{KO}$ in rodents and to allow for a finer control of genetic manipulation, an efficient way to manipulate gene expression is to use adeno-associated viruses (AAVs). An AAV-mediated genetic manipulation can be used to down- or upregulate a given molecular target and to restrict the manipulation to a specific cell population using different types of promoters ${ }^{14}$. AAVs are also extensively used as a delivery method in the clustered regularly interspaced short palindromic repeats (CRISPR)/ Cas9 technology 15,16 . These methodologies allow for better temporal and spatial control of genetic manipulation, which is generally associated with the expression of a reporter permitting quantification of the infected area using immunofluorescence.

AAVs also represent the main vector for cell typespecific manipulations of neuronal activity via optogenetics and chemogenetics ${ }^{17,18,19}$, which have been widely used in recent research on neurodegenerative diseases, behavior, cognition, and sleep $20,21,22$. In sleep research, the application of optogenetics for the activation or inhibition of certain brain regions, such as the basal forebrain, hypothalamus, and sublaterodorsal tegmentum, has been useful to determine their roles in the control of arousal, slow-wave sleep (also known as non-rapid eye movement sleep), paradoxical sleep (or rapid eye movement sleep), and cataplexy 23,24,25. Furthermore, AAVmediated manipulations have helped elucidate important sleep regulatory circuits and molecules contributing to the adverse effects of sleep loss $26,27,28$. For instance, one protein shown to be implicated in sleep deprivationinduced memory impairment is cofilin 29,30 . This protein is a filamentous actin-severing protein that participates in the reorganization of actin filaments by physically binding to actin and promoting the disassembly of the filaments in a dynamic manner ${ }^{31}$. Inhibiting cofilin activity using an AAV-mediated approach was shown to prevent spine loss as well as synaptic plasticity and memory deficits induced by sleep deprivation in mice $^{29}$. Collectively, these studies emphasize the usefulness and relevance of $\mathrm{AAV}$-mediated manipulations to understand sleep regulation and the consequences of sleep deprivation in rodents. 
Here, a protocol is described that combines ECoG and EMG electrode implantation and recording with the manipulation of cofilin function in a cerebral cortex area of wild-type (WT) mice using an AAV. More precisely, an AAV (serotype 9) expressing the coding sequence of a phosphomimetic form of the mouse cofilin (cofilin ${ }^{\mathrm{SD}}$ ), rendering it inactive ${ }^{32,33}$, is injected in the motor cortex (M1 and M2). An ECoG electrode is implanted directly at the injection site to ensure recording of the synchronized cortical activity of the infected cells. The ECoG/EMG recording is conducted for $24 \mathrm{~h}$ under undisturbed conditions three weeks after surgery to allow for recovery, adaptation, and high cofilin ${ }^{S 3 D}$ expression. The recording is then used for the identification of vigilance states and ECoG spectral analysis, as described in previous studies $^{11,34}$. This methodology can specifically reveal how cortical cofilin modulates wakefulness and sleep ECoG signals in mice. This combination of electrophysiological recordings and AAV-mediated genetic manipulation is particularly relevant to investigate the roles of various molecular elements in specific brain functions and could be applied to cortical (and subcortical) brain area(s) of interest in WT and genetically modified mice of both sexes and even other species.

\section{Protocol}

All methods were approved by the Comité d'éthique de l'expérimentation animale of the Recherche CIUSSS-NIM and are in accordance with guidelines of the Canadian Council on Animal Care. See the Table of Materials for reagents, equipment, and materials used in this protocol.

\section{Surgery preparation}

1. Preparation of ECoG and EMG electrodes
1. For each animal, prepare three ECoG electrodes: using a soldering iron, place a small drop of lead-free solder on the screw cap of a gold-covered screw, and solder a $4 \mathrm{~mm}$ long, $0.2 \mathrm{~mm}$ diameter gold wire (non-insulated) on the top of the screw cap using the lead-free solder (Figure 1A). Prepare 2 electrodes with the gold wire straight up, and one with a $45^{\circ}$ angle from the vertical.

2. For each animal, prepare two EMG electrodes: cut one $0.2 \mathrm{~mm}$ diameter gold wire to a length of 1.5 $\mathrm{cm}$ and a second one to $2 \mathrm{~cm}$. Curve both wires for these to embrace the curve of the skull up to the neck muscles, keeping a straight end that will be soldered to the connector (Figure 1B).

3. For each animal, prepare one connector: use a 6channel connector $(5 \mathrm{~mm} \times 8 \mathrm{~mm} \times 8 \mathrm{~mm}+3 \mathrm{~mm}$ metal pins), and add lead-free solder to 5 of the 6 metal pins (omitting one middle pin; Figure 1C). Cover the top of the connector with tape to avoid litter or water infiltration.

2. Preparation of AAVs and syringe pump

1. Prepare the test AAV (here, AAV9-CaMKIla0.4-

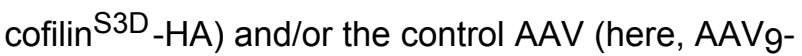
CaMKIla0.4-eGFP) by diluting the stock AAV $\operatorname{mix}(\mathrm{es})$ (here, AAVs in a solution of phosphatebuffered saline containing non-ionic surfactant $[0.001 \%])$ with sterile saline to obtain the desired viral titer (generally $10^{12-13}$ genome copies [GC]/ $\mathrm{mL}$ ) and the required volume for the number of mice to be treated.

NOTE: An injected volume of $1 \mu \mathrm{L}$ per cortical area per mouse requires the preparation of $2 \mu \mathrm{L}$. 
2. Fix one $10 \mu \mathrm{L}$ syringe to a syringe pump and fill it with distilled water.

3. Fill one PE50 tube of approximately $60-\mathrm{cm}$ length with distilled water using a $1 \mathrm{~mL}$ syringe and a $21 \mathrm{G}$ needle. Importantly, leave the $21 \mathrm{G}$ needle and syringe in place after filling. Connect the PE50 tube with the syringe pump; leave the $21 \mathrm{G}$ needle/ syringe on one end of the PE50 tube, and connect the other end to the $10 \mu \mathrm{L}$ syringe.

4. Once the tube is fixed to the $10 \mu \mathrm{L}$ syringe, remove the needle at the other end, and push the piston of the $10 \mu \mathrm{L}$ syringe to fill the gap left by the needle with water.

NOTE: Make sure there is no air bubble, and that the tube is completely filled with water.

5. Install a $28 \mathrm{G}$ cannula at the end of the tube where the needle was removed. Push water into the cannula with the $10 \mu \mathrm{L}$ syringe to fill it completely. Fix the cannula tightly to the stereotaxic arm.

3. Preparation of animals

NOTE: C57BL6/J male and female mice of $\sim 12$ weeks of age were previously adapted for at least 2 weeks to housing in individual cages and to a 12-h light:12-h dark cycle with ad libitum food and water and access to a wooden cube.

1. Carefully weigh the mice, and inject intraperitoneally a mix of ketamine/xylazine $(120 / 10 \mathrm{mg} / \mathrm{kg})$ for anesthesia. Wait approximately $10 \mathrm{~min}$ for deep anesthesia.

2. Shave the hair from the back of the ears to the front of the head between the eyes using a hair trimmer. NOTE: Be very careful not to cut the whiskers (protect the whiskers with a finger during shaving) as whisker trimming will modify sensory inputs and ECoG activity ${ }^{35,36}$.

3. Add a generous drop of ophthalmic ointment on each eye to prevent dehydration. Verify the depth of anesthesia regularly during the procedure by pinching a toe from the hind paw. Provide the mouse with $0.5-1.5 \%$ isoflurane to ensure deep anesthesia if a toe pinch reflex appears.

\section{Intracortical AAV injection with a syringe pump}

NOTE: Perform all the following steps with sterilized instruments and in a clean environment. Use $70 \%$ ethanol to further wash sterilized instruments and to wash electrodes prepared in section 1.1 as well as anchor screws (non-goldcovered screws) before beginning the surgery.

1. Carefully fix the head of the mouse on the stereotaxic apparatus with ear bars.

NOTE: Make sure the head is not moving laterally.

2. Gently pull the tongue of the animal out of the mouth to avoid suffocation, and fix the nose of the mouse with the stereotaxic adapter.

NOTE: Monitor the breathing frequently during the procedure.

3. Sterilize the shaved area of the head with $70 \%$ ethanol and by holding the skin with an extra fine Graefe forceps, cut the skin from the base of the ears to the level of the eyes with tissue scissors. Use four surgical clamps to stretch the skin and expose the skull (two on each side of the incision; see Figure 1D).

4. Scratch the skull surface with a sharp scissor tip: while avoiding bone sutures, remove the periosteum and create overlapping streaks in two or more directions. 
Remove the bone fragments, and dry the skull with $70 \%$ ethanol.

NOTE: The scratching and the streaking will help make the recording montage more robust by improving the adherence of the cement to the skull (see below).

5. With the cannula fixed to the stereotaxic arm, identify the location of the bregma (i.e., the intersection between the skull coronal and sagittal sutures; Figure 1D) and lambda (i.e., the intersection between the skull sagittal suture and a straight line connecting the left and right lambdoid suture; Figure 1D), and note the stereotaxic coordinates of each. If the difference between $z$ coordinates (vertical axis) of the bregma and lambda is greater than $0.3 \mathrm{~mm}$, adjust the height of the nose using the stereotaxic adapter until the $z$ position of bregma and lambda are aligned.

6. Mark the position of the cannula on the skull with a pen at these coordinates (motor cortex): $1.5 \mathrm{~mm}$ lateral right to midline and $1.5 \mathrm{~mm}$ anterior to bregma. Carefully pierce the skull at the position of the cannula with a $0.7 \mathrm{~mm}$ drill bit in a direction perpendicular to the skull surface (aligned with the vertical axis). Wash the pierced skull with a sterile cotton tip impregnated with a $10 \%$ providone-iodine solution.

7. Load the cannula with a $1 \mu \mathrm{L}$ air bubble by pulling the $10 \mu \mathrm{L}$ syringe piston back by $1 \mu \mathrm{L}$. Load the test AAV (here $A A V_{9}-C_{1}$ MKIla0.4-Cofilin ${ }^{S 3 D_{-}}-\mathrm{A}$ ) or the control AAV (here AAV9-CaMKIla0.4-eGFP) into the cannula previously loaded with the air bubble: mix the AAV by slowly pipetting up and down, pipet $1.7 \mu \mathrm{L}$ on a sterile Petri dish, and aspirate $1.5 \mu \mathrm{L}$ of the solution in the cannula by slowly pulling the piston of the $10 \mu \mathrm{L}$ syringe. Mark the position of the air bubble on the PE50 tube to allow the tracking of the injection.

8. Align the cannula with the hole on the skull for the vertical position of the cannula to reach the upper edge of the skull (i.e., skull surface). From the skull surface, slowly lower the cannula by $1.5 \mathrm{~mm}$ (to reach $1.5 \mathrm{~mm}$ below skull surface and layer $\mathrm{V}$ of the motor cortex).

NOTE: Be very careful not to lower the cannula too much to avoid unnecessary lesion of the brain tissue.

9. Start the syringe pump to inject $1 \mu \mathrm{L}$ of $\mathrm{AAV}$ over the course of $40 \mathrm{~min}$ (speed: $0.025 \mu \mathrm{L} / \mathrm{min}$ to minimize tissue damage). Keep track of the injection on the PE50 tube with the movement of the air bubble, and make adjustments if required.

10. After the injection is completed, leave the cannula in place for $5 \mathrm{~min}$ to ensure sufficient diffusion and avoid backflow. Then, slowly and carefully lift the stereotaxic arm to remove the cannula from the cortex.

\section{ECoG/EMG electrode implantation}

1. Using straight Kelly forceps, slowly screw one ECoG electrode (with straight gold-wire) in the vertical axis (same angle that the hole was pierced) in the hole where the AAV was injected. Leave at least $2.5 \mathrm{~mm}$ of the screw out of the skull to minimize damage to the dura and cerebral cortex (i.e., for an approximate depth of $1.1 \mathrm{~mm}$ from the skull surface; Figure 1D).

2. Mark the position of the posterior ECoG electrode and the reference electrode on the skull with a pen at these coordinates: posterior electrode (visual cortex) $1.5 \mathrm{~mm}$ lateral right to midline and $1.5 \mathrm{~mm}$ anterior to lambda, reference electrode (somatosensory cortex) $2.6 \mathrm{~mm}$ lateral right to midline and $0.7 \mathrm{~mm}$ posterior to bregma. 
Also, mark the position of three maintenance screws (acting as anchors between the skull and dental cement to solidify head montage) on the left hemisphere with no specific coordinates, but as distant as possible from each other and from the ECoG electrodes.

3. Carefully pierce the skull at the marked position of the other electrodes and anchor screws with the $0.7 \mathrm{~mm}$ drill bit. Pierce in a direction perpendicular to the skull surface for each screw (i.e., vertical axis for the posterior electrode but with an angle from the vertical axis for other sites). Wash the pierced skull with a $10 \%$ providoneiodine solution, and block the holes with small, rolled pieces of delicate task wipers before installing the screws to prevent bleeding and contamination.

4. Using the straight Kelly forceps, screw the maintenance screws in the left hemisphere and then screw the last two electrodes in the right hemisphere. Make sure to screw with the same angle that the holes were pierced and to leave at least $2.5 \mathrm{~mm}$ of each screw out of the skull (Figure 1D).

NOTE: To maximize the solidity of the final montage and the quality of the electrophysiological signals, be careful not to touch any screw when installing the next one.

5. Place a few small drops of dental cement in the center of the ring-like space inside the screws. Insert the curved end of one EMG electrode (prepared at step 1.1.2) approximately $1-2 \mathrm{~mm}$ in the neck muscles by holding the curved extremity using Dumont \#5 forceps and lifting the skin above muscles with extra-fine Graefe forceps. Then, place the curved side and elbow of the electrode in the dental cement; repeat for the second EMG electrode.

NOTE: The straight end of the longer EMG electrode should be aligned with the anterior ECoG electrode and that of the shorter EMG electrode with the posterior ECoG electrode. Make sure the two EMG electrodes are not touching each other or any of the screws.

6. Cover the eyes of the mice, and apply light for 3-5 min to help cement solidification. Once the EMG electrodes are holding firmly, cover the base of the ECoG electrodes and the base of the anchor screws with dental cement to form a crown-shaped contour. Cover the eyes of the mice, and apply light for 3-5 min to help cement solidification.

NOTE: Do not apply cement on the ECoG and EMG electrode extremities (gold wire that will be soldered to the connector) or on the skin.

7. Fill the center of the montage with previously mixed acrylic cement. During cement solidification, remove the four surgical clamps holding the skin (and wash these immediately with delicate task wipers).

NOTE: Do not apply cement on the ECoG and EMG electrode extremities (gold wire that will be soldered to the connector) or on the skin.

8. Suture the skin at the back and the front of the montage so that the skull is not exposed (but avoid stretching the skin too much) using a suture needle $(13 \mathrm{~mm} 3 / 8 \mathrm{c}$ ) and synthetic absorbable monofilament.

9. Hold the connector above the montage with curved forceps, and carefully align the gold wire of electrodes with the connector pins. Solder electrode extremities to connector pins with the soldering iron.

NOTE: Proceed quickly to avoid overheating and damage to the cortical tissue. Make sure that each electrode makes good contact with the corresponding connector pin, and that the electrodes are not connected to each other. 
10. Remove the mouse from the stereotaxic frame. Cover the empty space between the connector and the head with previously mixed acrylic cement by covering all connections between electrodes and connector pins.

NOTE: Avoid cement infiltration inside the connector by holding the mouse with the connector above the head (head straight not leaning).

11. Weigh the mouse and place it in a clean cage (preferably equipped with a non-meshed lid) on a heat pad (individual housing to avoid damage to the head montage). Monitor the animal regularly, and administer $0.1 \mathrm{mg} / \mathrm{kg}$ of buprenorphine subcutaneously upon awakening, and $12 \mathrm{~h}$ later if the animal shows signs of pain (e.g., abnormal posture, squinted eyes).

NOTE: Weight gain relative to pre-surgery weight should not exceed $1.5 \mathrm{~g}$.

\section{Recordings}

1. House mice individually to avoid damage to the head montage as a result of mutual grooming as well as damage and entanglement of the recording cables.

NOTE: For this protocol, mice were housed with ad libitum access to food, water, and a wooden cube, and daily monitoring was conducted.

2. Connect the mice to recording cables 2 weeks after surgery for adaptation to cabling conditions.

3. Record ECoG/EMG signals for $24 \mathrm{~h}$ (or longer/shorter depending on research questions).

NOTE: ECoG/EMG signal recording was accomplished using a cable, a swivel connector (to allow rotation of the cable), a 36-channel wearable box, and an amplifier, which was connected to a computer. Signals are sampled at $256 \mathrm{~Hz}$ (or more depending on research questions) and recorded with commercial software (see the Table of Materials). To ensure sufficient viral expression, experiments should be done at least 3 weeks after AAV injection, as described previously 29,37 .

4. After recording, sacrifice the mice by cervical dislocation (or other methods depending on the immunostaining protocol), and harvest the brain for immunostaining.

\section{Representative Results}

After electrophysiological recordings, immunofluorescence is used to define the area infected by the AAV injection and to validate the expression of cofilin ${ }^{S \mathrm{D}}$ (Figure 2). Immunostaining can be performed using a methodology similar to what has been described previously $29,37,38,39$. The AAV expresses an inactive form of cofilin fused with a

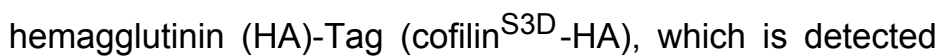
by immunofluorescence using an anti-HA antibody and a secondary antibody (Alexa Fluor 488). The infected excitatory neurons (here, targeted with a calcium/calmodulin-dependent protein kinase II alpha [CamKIla] promoter controlling the expression of the transgene contained in the $\mathrm{AAV}$ ) are stained with the anti-HA antibody. A successful infection is indicated by the staining of the neurons in the motor cortex surrounding the injection site (Figure 2A,B). In this representative example, the cerebral cortex of the other hemisphere did not show any noticeable staining. Nonetheless, given that excitatory neurons can project to distant brain areas, staining in the contralateral hemisphere is not necessarily an indication of unsuccessful injection. Higher magnification of the infected area showed staining of cell bodies and projections, confirming that only specific cells of the targeted cortical area were infected (Figure 2C).

Co-staining with markers of excitatory neurons (e.g., vesicular glutamate transporter 1 , CaMKIla) can also be performed 
to validate cell type-specificity. Alternatively, co-staining with markers of inhibitory neurons or astrocytes can be performed in case these cells are targeted using different promoters. Costaining of cofilin ${ }^{\mathrm{S} 3 \mathrm{D}_{-}}-\mathrm{HA}$ and CaMKIla was also performed in the same animal for an area more posterior to the injection site that still showed anti-HA staining in the motor cortex (Figure 2D). The higher magnification image of the area shows cells clearly expressing cofilin ${ }^{\mathrm{S} 3 \mathrm{D}_{-} \mathrm{HA}}$ (Alexa Fluor 488, Figure 2E) and CaMKIla (Alexa Fluor 568, Figure 2F). The superposition of the cofilin ${ }^{S 3 D_{-}}-\mathrm{A}$ and CaMKIla staining reveals that most (if not all) cells stained for cofilin ${ }^{S 3 D_{-}-H A}$ are also positive for CaMKIla (Figure 2G). This observation supports the specificity of the infection for excitatory neurons.

To assess the impact of cofilin manipulation on ECoG activity, ECoG and EMG signals are used to perform a visual identification of vigilance states (wakefulness, slow wave sleep, paradoxical sleep). This is done on 4-s epochs because of the rapid change in vigilance state in the mouse ${ }^{2}$, and here, for a full 24-h recording. Standard analyses include computation of sleep architecture and spectral analysis variables, as conducted previously for different datasets $^{11,12,13,28,34}$. In particular, spectral analysis of the ECoG signal of the different states will index state composition and quality. To remove differences that could arise, for instance, from different depths of the electrodes, spectral analysis data can be expressed relative to the total power of all states of a given animal (Figure 3A). Given the very low relative amplitude of ECoG activity in higher frequencies, relative power spectra for wakefulness, slow wave sleep, and paradoxical sleep have been logtransformed to more adequately visualize and simultaneously compare the activity in low and high frequencies. This analysis indicates state-specific differences in spectral activity under conditions of cofilin inactivation (Figure 3B). More precisely, these preliminary findings combining male and female mice point out that cofilin inactivation significantly increases spectral power in fast frequencies $(14-30 \mathrm{~Hz})$ during wakefulness and in slow frequencies (1-4 Hz) during paradoxical sleep, while leaving ECoG activity during slowwave sleep mainly unaffected. In addition, cofilin inactivation appears to increase inter-mouse variability in ECoG activity (particularly noticeable from error bars for wakefulness in Figure 3B). 

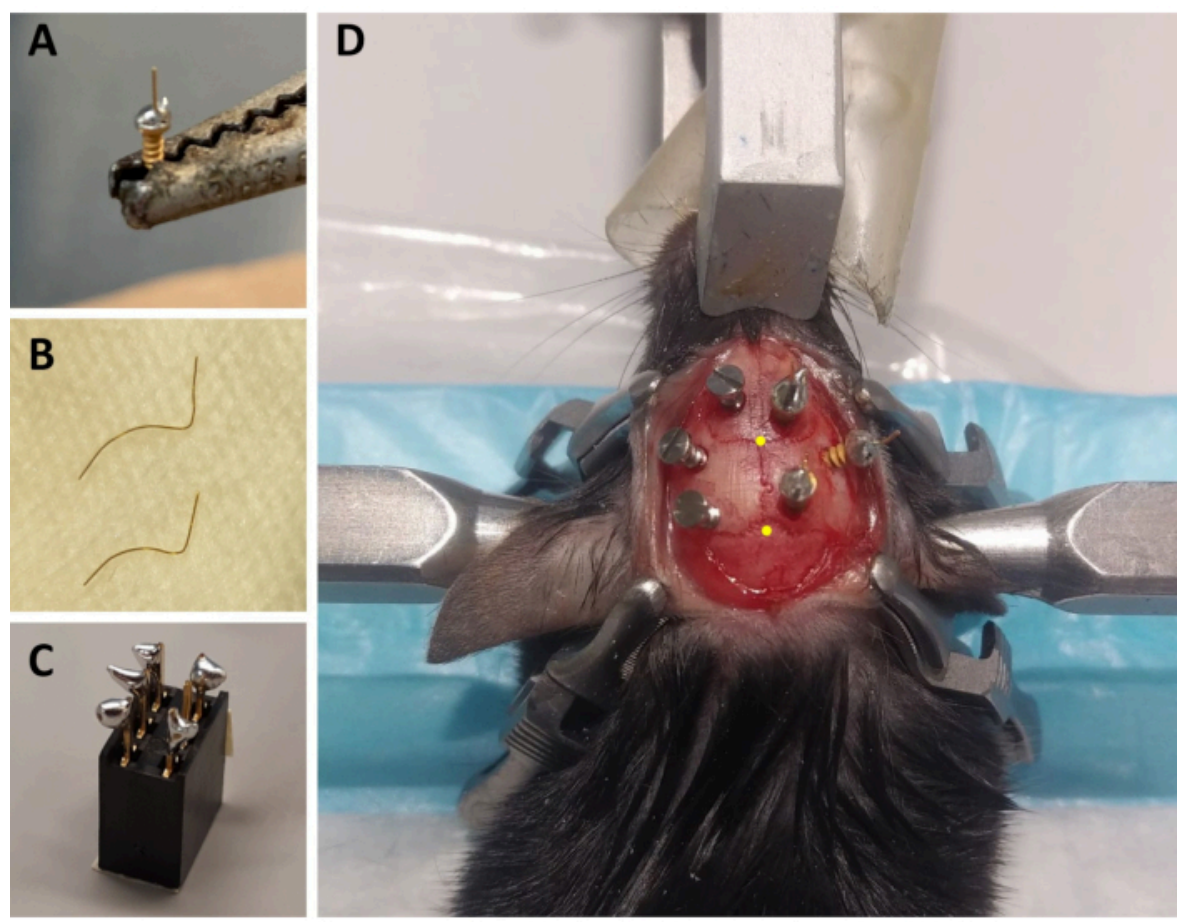

\section{Figure 1: Preparation of ECoG/EMG montage components and representative example of ECoG electrode}

placement. (A) An ECoG electrode: a $4 \mathrm{~mm}$ long, $0.2 \mathrm{~mm}$ diameter gold wire (non-insulated) is fused on the head of a gold-covered screw (1.9 mm head diameter, $1.14 \mathrm{~mm}$ thread major diameter, $3.6 \mathrm{~mm}$ total length) using lead-free solder. (B) EMG electrodes: two gold wires $(1.5$ and $2 \mathrm{~cm}$ ) are curved to embrace the curve of the skull up to the neck muscle, and the other end is kept straight to be soldered to the connector. (C) A 6-channel connector: lead-free solder is added to 5 of the 6 metal pins (omitting one in the middle) of the connector ( $5 \mathrm{~mm} \times 8 \mathrm{~mm} \times 8 \mathrm{~mm}+3 \mathrm{~mm}$ metal pins). The top of the connector is covered with tape to avoid litter/water infiltration. (D) Example of the positioning of the three maintenance screws on the skull of the left hemisphere and of the three ECoG electrodes (including a reference electrode) on the right hemisphere. The precise stereotaxic coordinates of the ECoG electrodes are indicated in steps 2.6 and 3.2 and have been calculated according to the location of the bregma and lambda (which are indicated by the yellow dots). Abbreviations: ECoG = electrocorticographic; EMG = electromyographic. Please click here to view a larger version of this figure. 

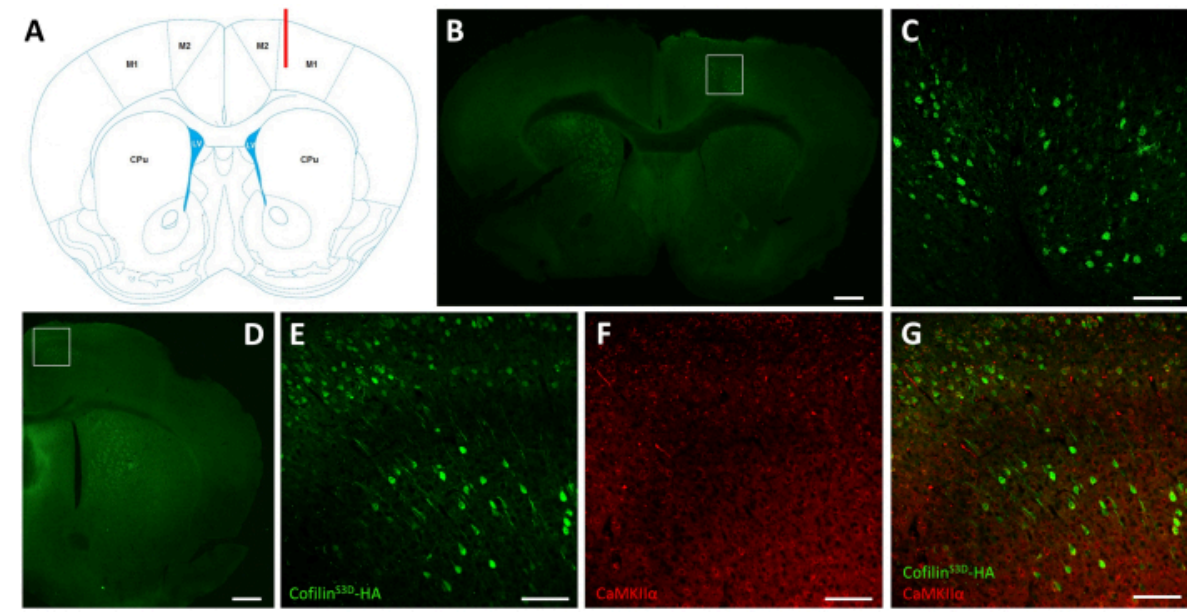

Figure 2: Representative immunostaining to define the AAV-infected area and cell type. (A) Schematic representation showing the injection site of the coronal slice presented in panel $B$. The position is $1.1 \mathrm{~mm}$ anterior to the bregma, and the cannula (shown in red) was targeted to layers $\vee$ of the right primary motor cortex (M1). Representation modified from Franklin and Paxinos ${ }^{40}$. (B) Immunostaining of HA to detect cofilin ${ }^{S 3 D_{-}}$A expression in neurons shown for a coronal slice of the full brain located approximately $1.1 \mathrm{~mm}$ anterior to the bregma. The infected area mainly localizes to layers $\mathrm{V}$ and $\mathrm{VI}$ (infragranular layers) of the right primary and secondary motor cortices (M1 and M2). Scale bar $=500 \mu \mathrm{m}$. The square represents the area shown in C. (C) Higher magnification of the infected area showing staining of infected cells and confirming expression of cofilin ${ }^{\mathrm{SD}_{-}}$-HA in deeper layers of the motor cortex. Scale bar $=100 \mu \mathrm{m}$. (D) Co-immunostaining of HA and CaMKIla to assess cell type-specificity shown for a coronal slice of the right hemisphere located approximately 0.5 $\mathrm{mm}$ anterior to bregma and therefore, posterior to the injection site (same mouse as in panels $\mathrm{B}$ and $\mathrm{C}$ ). The infected area localizes to motor cortices (M1 and mainly M2). Scale bar $=500 \mu \mathrm{m}$. The square represents the area shown in E, F, and G. (E) Higher magnification of the infected area showing staining of infected cells and confirming expression of cofilin $\mathrm{S} \mathrm{D}_{-\mathrm{HA}}$. Scale bar $=100 \mu \mathrm{m} .(\mathbf{F})$ Higher magnification of the infected area showing staining of CaMKIla-positive cells. Scale bar $=$ $100 \mu \mathrm{m}$. (G) Higher magnification of the infected area showing co-labeling of cofilin ${ }^{\text {SD }}-\mathrm{HA}$ and CaMKIla, confirming that infected cells are CaMKIla-positive. Scale bar $=100 \mu \mathrm{m}$. Abbreviations: AAV = adeno-associated virus; $\mathrm{M} 1=$ primary motor cortex; M2 = secondary motor cortex; $\mathrm{CPu}=$ caudate putamen (striatum); LV = lateral ventricle; HA= hemagglutinin; CamKIla = calcium/calmodulin-dependent protein kinase II alpha. Please click here to view a larger version of this figure. 

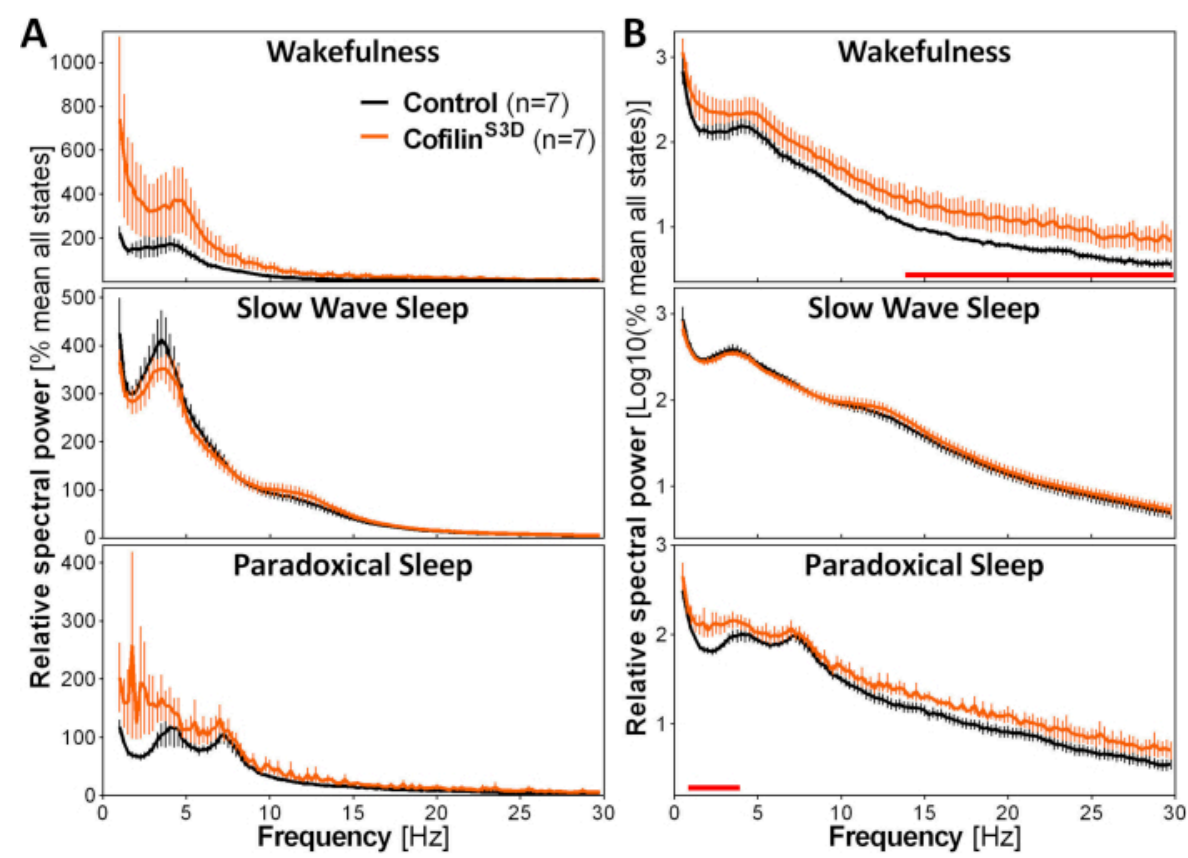

Figure 3: Representative power spectra for wakefulness, slow wave sleep, and paradoxical sleep obtained after viral manipulation of cofilin function. Male ( $n=5$ per group) and female ( $n=2$ per group) mice injected with AAV9-CaMKIla0.4-

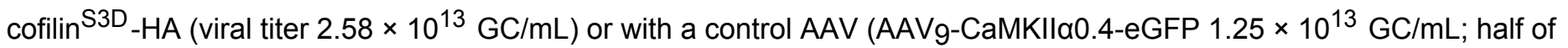
the test titer to control for the enhanced signal of this control $A A V$ ) in layer $V$ of the motor cortex were recorded for $24 \mathrm{~h}$, and the electrocorticographic signal was subjected to spectral analysis (fast Fourier Transform to calculate spectral power between 0.5 and $30 \mathrm{~Hz}$ with a $0.25-\mathrm{Hz}$ resolution). (A) Power spectra during the three vigilance states expressed relative to total power of all states. (B) Relative power spectra log-transformed to more adequately represent group differences in

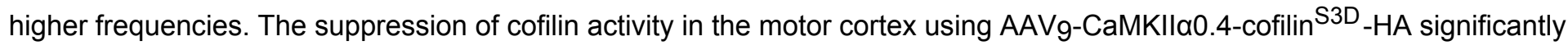
increases electrocorticographic activity in the beta range $(14-30 \mathrm{~Hz})$ during wakefulness, and in the delta range $(1-4 \mathrm{~Hz})$ during paradoxical sleep in comparison to control injections (red lines above $x$ axes indicate Mann-Whitney $U$-test on frequency band power $p<0.05)$. Abbreviations: $A A V=$ adeno-associated virus $G C=$ genome copies; $H A=$ hemagglutinin; CamKIl $\alpha=$ calcium/calmodulin-dependent protein kinase II alpha; eGFP = enhanced green fluorescent protein. Please click here to view a larger version of this figure.

\section{Discussion}

This protocol describes a precise and straightforward method to monitor ECoG and EMG activity during the manipulation of molecular targets using AAVs. For adequate between-group comparison, it is highly recommended to always plan surgical procedures (AAV injection and electrode implantation) on the same day for test and control animals, and to record their electrophysiological signals simultaneously. To obtain similar viral expression between the test and control animals, injecting the same viral titer is desirable. In the present case, 
viral titer of control AAV had been decreased to half of the test AAV to ensure similar viral expression. Experimenters should be very careful with measurements of stereotaxic coordinates to ensure low between-animal variability in brain area/cortical layer targeting. Additionally, given that the injection depth is calculated from the skull surface, and that skull thickness varies with age and sex, the placement of the cannula should always be verified using post-protocol histology or immunohistochemistry (e.g., Figure 2) to ensure adequate positioning/depth of injection, and the stereotaxic coordinates should be adjusted if necessary. Throughout the 40-min AAV injection, it is very important to monitor the injection speed to rapidly detect and correct potential issues such as pump blockage. Some experimental steps are also crucial to obtain optimal electrophysiological signals. For instance, do not overscrew during electrode implantation; screws should stick out of the skull by at least $2.5 \mathrm{~mm}$ to minimize damage to the cerebral cortex and the formation of a glial scar. Afterwards, it is also tremendously important to $\mathrm{i}$ ) avoid applying cement to the extremities of the electrodes, ii) ensure a rapid soldering of the electrodes to the connector, and iii) make sure that there is no contact between the electrodes.

The procedure presented here for ECoG and EMG recording is extremely well established, simple, and widely used to monitor wakefulness and sleep in mice $2,11,13,34$. Continuous ECoG and EMG recordings can be performed for several consecutive days (and even weeks) and generate a very rich dataset that can be used to perform several lines of analysis comprising variables related to wakefulness and sleep amount and architecture $2,11,12$ (e.g., time spent in different states per light and dark periods, number of episodes of each state, 24-h distribution of sleep), wakefulness and sleep spectral content ${ }^{34,41}$ (e.g., power in different frequency bands [similar to Figure 3], scale-free activity), and characteristics of individual waves ${ }^{42,43,44}$ (e.g., slow-wave amplitude and slope). When used in combination with AAVmediated molecular manipulations, an additional advantage is the avoidance of potential developmental compensation that can occur in transgenic animals. With practice, the whole procedure, including the 40-min AAV injection, can be performed in approximately $90 \mathrm{~min}$. Mortality rate should be (very) low as the surgery is minimally invasive.

The simultaneous use of ECoG/EMG recording and targeted manipulation with AAV offers a variety of other advantages and applications. For instance, the precision of stereotaxic targeting, when adequately performed, is very high and replicable and is useful to determine the specific role of a given brain region (and/or a cell type or a molecular element within the region) in the regulation of sleep or other physiological processes. Several different cortical areas can thus be easily targeted using adaptations of the current protocol. Moreover, target manipulations using AAVs could be directed to a cortical/subcortical area different from the ECoG recording sites. In such cases, the burr hole for AAV injection could be covered by a small glass coverslip fixed using dental cement (or bone wax). For enhanced specificity, the AAV construction often includes a promoter that allows targeted infection of a precise cell type ${ }^{14}$. A CamKIla promoter was used in the present protocol to specifically target excitatory pyramidal cells ${ }^{14,29,45}$ of the motor cortex. This strategy has enabled the inactivation of cofilin (using cofilin $\left.^{\mathrm{S} 3 \mathrm{D}}\right)^{32,33}$ in excitatory neurons of the motor cortex and the observation of state-specific changes in ECoG activity (Figure 3). To assess infection/transduction efficacy, future protocol users could combine the presented AAV-ECoG protocol with one of co-staining by immunofluorescence, and use high magnification images to calculate the number of cells showing double-labeling out of the total number of 
cells showing single-labeling of the target (here, CaMKIlaexpressing neurons). In a recent study, an AAV-ECoG method similar to the one described here was used to overexpress fragile $X$ mental retardation syndrome-related protein 1 (FXR1) in all neurons of the motor cortex using an AAV containing a synapsin promoter and revealed an effect of this manipulation on vigilance state distribution and spectral content $^{28}$. These findings illustrate how manipulating a given molecule in a target brain region using AAVs can reveal roles in the regulation of specific wakefulness/sleep parameters.

A limitation of the described protocol is the small lesion of brain tissue occurring with cannula placement before performing the AAV injection, which could also be accompanied by an inflammatory response. This could be of particular concern when performing AAV injection in subcortical areas and should always be tackled by using adequate controls. Alternatively, the current protocol could be followed by the quantification of reactive gliosis and/or of microglial activation (e.g., using immunofluorescence) to ensure similar levels in control and test groups and therefore, on the $E C o G$ readout. $A$ second limitation relates to the risk of bad connection between an electrode and the connector, which could result in a continuously or occasionally bad electrophysiological signal. Solidly screwed, soldered, and cemented electrodes will minimize the incidence of this issue. A third limitation is related to animals being tethered via the head montage during the recording, which could limit locomotion and other behaviors, at least to some extent, and occasionally result in cabling damage and signal loss. Finally, the presented protocol is more suitable for adult mice, given that the skull size of younger animals may cause difficulties in installing the depicted head montage, as described previously ${ }^{2}$.
Combined ECoG/EMG recording and AAV-mediated manipulation of a precise target is also applicable to research fields other than the neuroscience of sleep. Among others, it could be used to study and manipulate epileptic events in animal models of seizure and is a powerful tool to modulate brain oscillations involved in memory encoding and consolidation 46,47 . Accordingly, potential applications certainly encompass the fields of fundamental research in psychiatry and neurology, including neurodegenerative diseases. In addition to the capacity of expressing an inactive form of a molecule, AAVs can and have been used to overexpress or downregulate (e.g., small-interfering RNA, CRISPR/Cas9) or to rescue the expression of a molecule in a full-body KO. Importantly, the dual methodology of the current protocol is also applicable to other mammalian species such as rats and diurnal rodents that represent interesting models to understand both sleep and neurodegeneration 48,49 .

\section{Disclosures}

The authors have nothing to disclose.

\section{Acknowledgments}

The work was funded by the Canada Research Chair in Sleep Molecular Physiology. The authors are thankful to Chloé Provost and Caroline Bouchard for technical help.

\section{References}

1. Campbell, I. G. EEG recording and analysis for sleep research. Current Protocols in Neuroscience. Chapter 10 (unit 10.2) (2009).

2. Mang, G. M., Franken P. Sleep and EEG phenotyping in mice. Current Protocols in Mouse Biology. 2 (1), 55-74 (2012). 
3. Bastien, C.H. et al. Insomnia and sleep misperception. Pathologie Biologie. 62 (5), 241-251 (2014).

4. Malafeev, A. et al. Automatic artefact detection in single-channel sleep EEG recordings. Journal of Sleep Research. 28 (2), e12679 (2019).

5. Latreille, V. et al. Electroencephalographic prodromal markers of dementia across conscious states in Parkinson's disease. Brain. 139 (Pt 4), 1189-1199 (2016).

6. Rodrigues Brazete, J. et al. Electroencephalogram slowing predicts neurodegeneration in rapid eye movement sleep behavior disorder. Neurobiology of Aging. 37, 74-81 (2016).

7. Kent, B. A., Strittmatter, S. M., Nygaard, H. B. Sleep and EEG power spectral analysis in three transgenic mouse models of Alzheimer's disease: APP/PS1, 3xTgAD, and Tg2576. Journal of Alzheimers Disease. 64 (4), 1325-1336 (2018).

8. Chang, A. M. et al. Circadian gene variants influence sleep and the sleep electroencephalogram in humans. Chronobiology International. 33 (5), 561-573 (2016).

9. Shi, G., Wu, D., Ptacek, L. J., Fu, Y. H. Human genetics and sleep behavior. Current Opinion in Neurobiology. 44, 43-49 (2017).

10. Nakai, Y. et al. Calcineurin and its regulator sra/ DSCR1 are essential for sleep in Drosophila. Journal of Neuroscience. 31 (36), 12759-12766 (2011).

11. El Helou, J. et al. Neuroligin-1 links neuronal activity to sleep-wake regulation. Proceedings of the National Academy of Sciences of the United States of America. 110 (24), 9974-9979 (2013).
12. Freyburger, M. et al. EphA4 is involved in sleep regulation but not in the electrophysiological response to sleep deprivation. Sleep. 39 (3), 613-624 (2016).

13. Seok, B. S. et al. The effect of Neuroligin-2 absence on sleep architecture and electroencephalographic activity in mice. Molecular Brain. 11 (1), 52 (2018).

14. Haery, L. et al. Adeno-associated virus technologies and methods for targeted neuronal manipulation. Frontiers in Neuroanatomy. 13, 93 (2019).

15. Schmidt, F., Grimm, D. CRISPR genome engineering and viral gene delivery: a case of mutual attraction. Biotechnology Journal. 10 (2), 258-272 (2015).

16. Wang, D., Zhang, F., Gao, G. CRISPR-based therapeutic genome editing: strategies and in vivo delivery by AAV vectors. Cell. 181 (1), 136-150 (2020).

17. Havekes, R. et al. Transiently increasing cAMP levels selectively in hippocampal excitatory neurons during sleep deprivation prevents memory deficits caused by sleep loss. Journal of Neuroscience. 34 (47), 15715-15721 (2014).

18. Fuller, P. M., Yamanaka, A., Lazarus, M. How genetically engineered systems are helping to define, and in some cases redefine, the neurobiological basis of sleep and wake. Temperature (Austin). 2 (3), 406-417 (2015).

19. Naso, M. F., Tomkowicz, B., Perry, W. L.3 ${ }^{\text {rd }}$, Strohl W. R. Adeno-associated virus (AAV) as a vector for gene therapy. BioDrugs. 31 (4), 317-334 (2017).

20. Ono, D., Yamanaka, A. Hypothalamic regulation of the sleep/wake cycle. Neuroscience Research. 118, 74-81 (2017). 
21. Shiromani, P. J., Peever, J. H., New neuroscience tools that are identifying the sleep-wake circuit. Sleep. 40 (4), zsx032 (2017).

22. Oishi, N. et al. Artificial association of memory events by optogenetic stimulation of hippocampal CA3 cell ensembles. Molecular Brain. 12 (1), 2 (2019).

23. Anaclet, C. et al. Genetic activation, inactivation, and deletion reveal a limited and nuanced role for somatostatin-containing basal forebrain neurons in behavioral state control. Journal of Neuroscience. 38 (22), 5168-5181 (2018).

24. Chen, K.S. et al. A hypothalamic switch for REM and nonREM sleep. Neuron. 97 (5), 1168-1176.e4 (2018).

25. Torontali, Z. A., Fraigne, J. J., Sanghera, P., Horner, R., Peever, J. The sublaterodorsal tegmental nucleus functions to couple brain state and motor activity during REM sleep and wakefulness. Current Biology. 29 (22), 3803-3813.e5 (2019).

26. Rolls, A. et al. Optogenetic disruption of sleep continuity impairs memory consolidation. Proceedings of the National Academy of Sciences of the United States of America. 108 (32), 13305-13310 (2011).

27. Ognjanovski, N., Broussard, C., Zochowski, M., Aton, S. J. Hippocampal network oscillations rescue memory consolidation deficits caused by sleep loss. Cerebral Cortex. 28 (10), 3711-3723 (2018).

28. Khlghatyan, J. et al. Fxr1 regulates sleep and synaptic homeostasis. EMBO Journal. 39 (21), e103864 (2020).

29. Havekes, R. et al. Sleep deprivation causes memory deficits by negatively impacting neuronal connectivity in hippocampal area CA1. Elife. 5, e13424 (2016).
30. Wong, L. W., Tann, J. Y., Ibanez, C. F., Sajikumar, S. The p75 neurotrophin receptor is an essential mediator of impairments in hippocampal-dependent associative plasticity and memory induced by sleep deprivation. Journal of Neuroscience. 39 (28), 5452-5465 (2019).

31. Mizuno, K. Signaling mechanisms and functional roles of cofilin phosphorylation and dephosphorylation. Cellular Signalling. 25 (2), 457-469 (2013).

32. Nagaoka, R., Abe, H., Obinata T. Site-directed mutagenesis of the phosphorylation site of cofilin: its role in cofilin-actin interaction and cytoplasmic localization. Cell Motility and the Cytoskeleton. 35 (3), 200-209 (1996).

33. Elam, W.A. et al. Phosphomimetic S3D cofilin binds but only weakly severs actin filaments. Journal of Biological Chemistry. 292 (48), 19565-19579 (2017).

34. Areal, C. C., Cao, R., Sonenberg, N., Mongrain, V. Wakefulness/sleep architecture and electroencephalographic activity in mice lacking the translational repressor 4E-BP1 or 4E-BP2. Sleep. 43 (2), ZSZ210 (2020).

35. Vyazovskiy, V., Borbely, A.A., Tobler, I. Unilateral vibrissae stimulation during waking induces interhemispheric EEG asymmetry during subsequent sleep in the rat. Journal of Sleep Research. 9 (4), 367-371 (2000).

36. Sitnikova, E. Neonatal sensory deprivation promotes development of absence seizures in adult rats with genetic predisposition to epilepsy. Brain Research. 1377, 109-118 (2011). 
37. Tudor, J. C. et al. Sleep deprivation impairs memory by attenuating mTORC1-dependent protein synthesis. Science Signaling. 9 (425), ra41 (2016).

38. Evilsizor, M. N., Ray-Jones, H. F., Lifshitz, J., Ziebell, J. Primer for immunohistochemistry on cryosectioned rat brain tissue: example staining for microglia and neurons. Journal of Visualized Experiments. (99), e52293 (2015).

39. Dufort-Gervais, J. et al. Neuroligin-1 is altered in the hippocampus of Alzheimer's disease patients and mouse models, and modulates the toxicity of amyloid-beta oligomers. Scientific Reports. 10 (1), 6956 (2020).

40. Franklin, K. B. J., Paxinos, G. The mouse brain in stereotaxic coordinates, Third edition. Academic Press, New York (2007).

41. Lina, J. M., O'Callaghan, E. K., Mongrain, V. Scale-free dynamics of the mouse wakefulness and sleep electroencephalogram quantified using WaveletLeaders. Clocks \& Sleep. 1 (1), 50-64 (2019).

42. Massart, R. et al. The genome-wide landscape of DNA methylation and hydroxymethylation in response to sleep deprivation impacts on synaptic plasticity genes. Translational Psychiatry. 4, e347 (2014).

43. Freyburger, M., Poirier, G., Carrier, J., Mongrain, V. Shorter duration of non-rapid eye movement sleep slow waves in EphA4 knockout mice. Journal of Sleep Research. 26 (5), 539-546 (2017).

44. Hubbard, J. et al. Rapid fast-delta decay following prolonged wakefulness marks a phase of wake-inertia in NREM sleep. Nature Communications. 11 (1), 3130 (2020).

45. Johansen, J. P. et al. Optical activation of lateral amygdala pyramidal cells instructs associative fear learning. Proceedings of the National Academy of Sciences of the United States of America. 107 (28), 12692-12697 (2010).

46. Boyce, R., Glasgow, S. D., Williams, S., Adamantidis, A. Causal evidence for the role of REM sleep theta rhythm in contextual memory consolidation. Science. 352 (6287), 812-816 (2016).

47. Bandarabadi, M. et al. Dynamic modulation of thetagamma coupling during rapid eye movement sleep. Sleep. 42 (12), zsz182 (2019).

48. Estrada, C. et al. Transcranial magnetic stimulation and aging: Effects on spatial learning and memory after sleep deprivation in Octodon degus. Neurobiology of Learning and Memory. 125, 274-281 (2015).

49. Hurley, M. J. et al. The long-lived Octodon degus as a rodent drug discovery model for Alzheimer's and other age-related diseases. Pharmacology \& Therapeutics. 188, 36-44 (2018). 